\title{
The contribution of ophthalmology journals to undergraduate medical education
}

Eye (2017) 31, 981; doi:10.1038/eye.2017.17; published online 17 March 2017

The Internet has enabled global distribution of medical education and is increasingly replacing traditional textbooks. We investigated whether ophthalmology journals publish papers partly or primarily directed at undergraduates. In September 2015, we searched OVIDSP-Medline for each of the 56 ophthalmology journals listed by Thomson-Reuters for publications with 'undergraduate*' or 'medical student ${ }^{* \prime}$ in the title or abstract. We selected papers focussing mostly on or including a substantially relevant section about the education of medical undergraduates. We excluded papers on postgraduate education, selection into training, or perception of the specialty among students.

We found 168 publications, of which $78(46 \%)$ met the criteria for inclusion. Of 56 journals, 21 $(37.5 \%)$ published at least one paper meeting our search criteria. The number of publications has grown steadily in recent years, with 31 from the last 5 years. The majority originated from the US and Canada $(n=44)$ and from Europe $(n=15)$.

Publishing medical education papers in specialist journals is important for many reasons. For ophthalmology to continue to appeal widely to the best applicants, there must be adequate exposure for all students, not just those who realise its possibilities early on. There is pressure to make career choices earlier than ever, with applicants who do so being at a significant advantage over their peers at demonstrating their commitment. Increasing the profile of ophthalmology among medical students will pay dividends by improving motivation to learn about the eye and pick up practical skills. This need has been identified in other specialties with limited exposure in the medical student curriculum, such as maxillofacial surgery. ${ }^{1}$

For students who are particularly motivated to explore the specialty, there may be opportunities to include them in a study as an inexperienced control, for example, when testing the most reliable way to quickly learn a specialist skill such as gonioscopy. ${ }^{2}$

Medical students have free access to electronic journals through their medical school. We suggest specialty journals will increasingly form a point of reference for their studies and career explorations, and it is important that students' attempts to engage are provided for. We believe publication of papers targeted wholly or partly at undergraduates should be further encouraged.

\section{Conflict of interest}

The authors declare no conflict of interest.

\section{Acknowledgements}

We would like to thank Professor Lightman for her comments on our initial draft.

\section{References}

1 Ologunde R, Sykes M. A review of oral and maxillofacial surgery journals' contribution to undergraduate surgical education. Br J Oral Maxillofac Surg 2014; 52(10): 894-900.

2 Congdon NG, Spaeth GL, Augsburger J, Klancnik Jr J, Patel K, Hunter DG. A proposed simple method for measurement in the anterior chamber angle: biometric gonioscopy. Ophthalmology 1999; 106(11): 2161-2167.

\section{Barai ${ }^{1}$ and VA Nowak ${ }^{2}$}

${ }^{1}$ Faculty of Medicine, Imperial College London South Kensington Campus, London, UK

${ }^{2}$ Department of Ophthalmology Chelsea and Westminster Hospital NHS Foundation Trust, London, UK

Correspondence: I Barai, Faculty of Medicine, Imperial College, London South Kensington Campus, London SW7 2AZ, UK Tel: 07535659331. E-mail: Ishani.barai11@imperial.ac.uk 